\title{
ZEÓLITA 4A PARA PURIFICAÇÃo DO GÁS DE ATERRO SANITÁRIO
}

José D. V. de Souza Filho ${ }^{a}$, Ari C. A. de Lima ${ }^{a}$, Ronaldo Stefanuttia, Wildson M. B. da Silva ${ }^{\mathrm{b}}$, Moisés Bastos-Neto ${ }^{\mathrm{c}}$, Enrique Vilarrasa-Garciac ${ }^{c}$ Adonay R. Loiola ${ }^{\mathrm{b}, *}$ e Francisco S. B. Mota ${ }^{\mathrm{a}}$

a Departamento de Engenharia Hidráulica e Ambiental, Universidade Federal do Ceará, 60440-900 Fortaleza - CE, Brasil

bDepartamento de Química Orgânica e Inorgânica, Universidade Federal do Ceará, 60440-900 Fortaleza - CE, Brasil

'Departamento de Engenharia Química, Universidade Federal do Ceará, 60440-900 Fortaleza - CE, Brasil

Recebido em 14/05/2017; aceito em 15/08/2017; publicado na web em 16/10/2017

\begin{abstract}
ZEOLITE 4A FOR CLEANSING OF LANDFILL GAS. The anaerobic decomposition in landfills produces a gas mixture known as landfill biogas. This biogas consists mainly of $\mathrm{CH}_{4}$ and $\mathrm{CO}_{2}$. The $\mathrm{CO}_{2}$ removal can be carried out by means of adsorption. In order to promote $\mathrm{CO}_{2}$ removal, zeolite type $4 \mathrm{~A}$ was used as solid adsorbent. The synthesized zeolite was characterized by scanning electron microscopy and X-ray diffraction. $\mathrm{CH}_{4} / \mathrm{CO}_{2}$ mixture isotherms were obtained in a magnetic suspension balance equipped with a gas dosing unit and Extended Langmuir and Extended Sips models were used to predict mixture isotherms from monocomponent data. Three biogas emissions drains in a sanitary landfill in Caucaia (Ceará State, Brazil) - ASMOC - were chosen to test the performance of zeolite as $\mathrm{CO}_{2}$ adsorbent under real conditions. Zeolite $4 \mathrm{~A}$ has proven to be very selective for $\mathrm{CO}_{2}$ even in the presence of $\mathrm{H}_{2} \mathrm{~S}$.
\end{abstract}

Keywords: biogas; $\mathrm{CO}_{2}$ adsorption; zeolite $4 \mathrm{~A}$; separation; solid waste.

\section{INTRODUÇÃO}

Uma parte considerável dos resíduos sólidos urbanos é formada por materiais orgânicos e biodegradáveis. Após processos microbiológicos várias substâncias como metano $\left(\mathrm{CH}_{4}\right)$, dióxido de carbono $\left(\mathrm{CO}_{2}\right)$, ácido sulfídrico $\left(\mathrm{H}_{2} \mathrm{~S}\right)$ e ácidos graxos voláteis são geradas. $\mathrm{O}$ biogás de aterros sanitários, comumente chamado de gás de aterro, é o produto gasoso resultante da decomposição da matéria orgânica nos aterros sanitários. Com o passar do tempo, a matéria orgânica ali depositada sofre decomposição por meio de hidrólise, acidogênese, acetogênese e metanogênese. Desta última provém a formação do gás metano, cuja aplicação notadamente para fins industriais mostra-se viável. ${ }^{1} \mathrm{O}$ biogás de aterro consiste em uma mistura composta por $\mathrm{CH}_{4}\left(45-55 \%\right.$ v/v), $\mathrm{CO}_{2}\left(30-35 \%\right.$ v/v), $\mathrm{H}_{2}$ (cerca de $4 \%$ v/v), $\mathrm{NH}_{3}$ (em torno de $0,5 \% \mathrm{v} / \mathrm{v}$ ) além de $\mathrm{H}_{2} \mathrm{~S}$, ácidos graxos voláteis (AGV) e siloxisanos em teores menores. ${ }^{2}$

Para que o metano do biogás de aterro possa ser utilizado como combustível é imprescindível tratamento prévio com vistas à remoção de $\mathrm{CO}_{2}$, uma vez que este último compromete a capacidade calorífica do metano além de contribuir diretamente para a promoção de corrosão das tubulações dos sistemas de transporte e armazenamento na presença de umidade. ${ }^{3}$ Os principais métodos de separação de misturas $\mathrm{CH}_{4} / \mathrm{CO}_{2}$ incluem processos de separação por membranas, coluna de absorção e PSA (Pressure Swing Adsorption). ${ }^{3}$ Os processos adsortivos apresentam a vantagem da regulação da seletividade de acordo com os tipos e formas dos adsorventes usados nos processos de separação, o que constitui um diferencial frente a outras técnicas de separação. ${ }^{4} \mathrm{~A}$ adsorção em materiais porosos é energeticamente eficiente e competitiva do ponto de vista econômico, especialmente em casos onde volumes relativamente pequenos de gases necessitam serem processados. ${ }^{3}$ Dentre os materiais comumente usados para esta finalidade, destacam-se sílicas, ${ }^{3}$ carbonos ativados, ${ }^{4}$ argilas, ${ }^{5} \mathrm{e}$ zeólitas. ${ }^{5}$ Neste contexto, destacam-se as zeólitas, as quais podem ser empregadas em inúmeros processos em que se visa a remoção de $\mathrm{CO}_{2}$ em misturas gasosas, ${ }^{6}$ além da alta seletividade comumente observada comparada a outros métodos de separação. ${ }^{5}$

\footnotetext{
*e-mail: adonay@ufc.br
}

A técnica PSA tem sido bastante empregada em estudos de separação de misturas $\mathrm{CH}_{4} / \mathrm{CO}_{2}$ por meio de materiais nanoporosos, indicando a viabilidade do metano de biogás de aterro sanitário no que tange ao aproveitamento energético deste gás. ${ }^{8-10}$ Zeólitas são sólidos cristalinos microporosos à base de sílica, onde alguns átomos de silício podem ser substituídos por outros elementos tais como Al (principalmente), P, Fe, B, Ga, Ti, Ge etc. As zeólitas são caracterizadas por uma estrutura tridimensional resultante das ligações entre as unidades tetraédricas $\mathrm{TO}_{4}(\mathrm{~T}=\mathrm{Si}, \mathrm{Al}, \mathrm{P}, \mathrm{Fe}$ etc. $)$, as quais compartilham átomos de oxigênio nos vértices. Tal estrutura apresenta sistemas de canais e cavidades ordenados cujas dimensões permitem a adição e remoção de moléculas hospedeiras reversivelmente, tornando-as excelentes adsorventes. ${ }^{11,12}$ A zeólita 4A (tipo LTA) apresenta íons sódio que atuam como compensadores da carga negativa resultante da presença de átomos de alumínio tetraédrico na estrutura zeolítica. Seus poros principais, os quais apresentam abertura na ordem de $4 \AA$, conferem à zeólita elevado potencial para ser utilizada na purificação do biogás de aterros sanitários, com vistas a se obter metano com grau de pureza aceitável. ${ }^{13}$ Neste trabalho, o desempenho da zeólita $4 \mathrm{~A}$, sintetizada via rota hidrotérmica, na remoção do $\mathrm{CO}_{2}$ do gás foi estudado em laboratório e em um aterro sanitário, localizado na cidade de Caucaia - CE.

\section{PARTE EXPERIMENTAL}

\section{Síntese da zeólita 4A}

A zeólita 4A foi preparada de acordo com a metodologia descrita por Thompson et al. ${ }^{14}$ Em um béquer de polipropileno foram adicionados de 5,00 $\mathrm{g}$ de aluminato de sódio (Sigma-Aldrich) e 35 $\mathrm{mL}$ de hidróxido de sódio (Sigma-Aldrich) $0,21 \mathrm{~mol} \mathrm{~L}^{-1}$. Em outro béquer de polipropileno, foram misturados $7,16 \mathrm{~g}$ de metassilicato de sódio (Sigma-Aldrich) e $35 \mathrm{~mL}$ de hidróxido de sódio $0,21 \mathrm{~mol} \mathrm{~L}^{-1}$.

As soluções foram misturadas e transferidas para um cadinho de Teflon, o qual foi confinado em autoclave de aço inox. A mistura permaneceu em repouso por $18 \mathrm{~h}$ e em seguida foi submetida a aquecimento de $100^{\circ} \mathrm{C}$, em estufa, durante $4 \mathrm{~h}$. Após o resfriamento, a amostra foi lavada com água destilada oito vezes e seca a $80{ }^{\circ} \mathrm{C}$ 
durante $12 \mathrm{~h}$. A granulometria foi padronizada em $74 \mathrm{~mm} \cdot{ }^{14}$

A zeólita obtida foi moldada na forma de pellets. A preparação destes foi realizada por meio da maceração da zeólita na presença de uma pequena quantidade de solução aquosa de álcool polivinílico (PVA) $10 \% \mathrm{~m} / \mathrm{v}$. Com o auxílio de uma seringa, a mistura foi gotejada sobre uma placa de vidro. Os pellets foram secos em estufa a $50{ }^{\circ} \mathrm{C}$ por 30 minutos e, em seguida, calcinados a $550^{\circ} \mathrm{C}$ durante $2 \mathrm{~h}$, usando taxa de aquecimento de $5{ }^{\circ} \mathrm{C} \mathrm{min}^{-1}$.

\section{Caracterização da zeólita}

A zeólita 4A sintetizada foi caracterizada por meio de difração de raios-X (DRX) e microscopia eletrônica de varredura (MEV). Medidas de DRX foram realizadas em difratômetro para amostras policristalinas Panalytical modelo X-Pert PRO, com spinner e geometria Bragg-Brentano, operando em modo contínuo com radiação de $\mathrm{Cu} \mathrm{K} \alpha$ a $40 \mathrm{kV}$ e $45 \mathrm{~mA}$. O difratograma foi obtido no intervalo $2 \theta$ de 05 a $50^{\circ}$ e tempo de contagem de $67 \mathrm{~s}$. A identificação de fases cristalinas na amostra foi feita utilizando o aplicativo X-Pert HighScore.

Análises de MEV foram realizadas utilizando equipamento $F E G$, modelo Quanta 450. A amostra foi preparada em fita de carbono de dupla face sobre suporte de alumínio, sendo metalizada com ouro em atmosfera de argônio a baixa pressão, em equipamento Quorum, modelo Q15DT ES.

\section{Ensaio experimental de adsorção (Gravimetria)}

Foram obtidas as isotermas de $\mathrm{CH}_{4}$ puro, de $\mathrm{CO}_{2}$ puro e de mistura $50 \% \mathrm{CO}_{2} / 50 \% \mathrm{CH}_{4}(\mathrm{v} / \mathrm{v})$ a $40{ }^{\circ} \mathrm{C}$ até 10 bar numa balança de suspensão magnética, equipada com unidade de mistura, da marca Rubotherm ${ }^{\circledR}$. Antes dos testes de equilíbrio, a amostra foi regenerada a temperatura constante de $300^{\circ} \mathrm{C}\left(2^{\circ} \mathrm{C}\right.$ min $\left.^{-1}\right)$. A determinação dos dados de equilíbrio consiste em, após regeneração, submeter a amostra a incrementos sucessivos de pressão, em cada um dos quais são quantificadas alterações de massa da amostra (resolução de 0,01 mg) com relação à pressão, até o equilíbrio ser atingido.

A variação de massa registrada pela balança se dá como descrito pela Equação 1:

$$
\Delta m=m_{e x c}(P, T)-\left(V_{B}+V_{S}\right) \rho(P, T)
$$

Em que $\Delta m$ é a variação da massa registrada, $m_{e x c}$ é a massa adsorvida em excesso, $V_{B}+V_{S}$ é a soma dos volumes dos componentes da balança e o volume específico do sólido, respectivamente, e $\rho$ é a densidade do gás nas condições especificas de pressão e temperatura.

Não foram realizadas correções dos valores de massa em excesso por valores absolutos porque, na faixa de pressão em que foram feitos os testes, as desvios de quantidades absolutas e quantidades em excesso são negligenciáveis. ${ }^{15}$

\section{Ensaio experimental de adsorção (Leito fixo)}

\section{Preparação dos cartuchos}

Foram preparados cartuchos em cilindros de quartzo $(8 \mathrm{~mm} \mathrm{x}$ $100 \mathrm{~mm}$ ) contendo diferentes massas de zeólitas na forma de pellets (100 mg, 200 mg e 400 mg), como ilustrado na Figura 1, sendo que para cada massa utilizada foram preparados três cartuchos para a

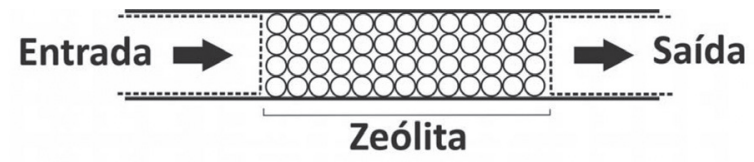

Figura 1. Esquematização de cartucho com zeólitas empacotadas realização dos ensaios de adsorção em triplicata. Nas extremidades de cada cartucho foi conectada a mangueira de sucção do equipamento de medição de gás de aterro, o GEM 5000, da Landtech ${ }^{\circledR}$.

\section{Medições de biogás no aterro}

O equipamento GEM 5000 da Landtech $^{\circledast}$ possui uma zona de entrada (inlet) e uma zona de saída (outlet). Ao ser ligado, o GEM aciona uma bomba de sucção na zona de entrada e, assim, aduz o gás de aterro para medição. Cada cartucho com zeólitas, devidamente preparado, foi conectado à zona de saída do GEM. Antes de passar pelo inlet, o biogás atravessa um filtro em formato de disco com porosidade de 0,2 mm (Midisart $^{\circledR}$ ). Em seguida, o biogás filtrado é sugado pelo GEM e lançado pelo outlet. Nesta saída, uma mangueira é conectada a uma extremidade de um cartucho. A pressão do outlet força o biogás a passar pelo cartucho e, assim, facilitando o contato do biogás com a zeólita. Os valores de captação do $\mathrm{CO}_{2}$ pelo $G E M$ ocorreram em tempo real. As condições de operação foram de 1 bar e $40{ }^{\circ} \mathrm{C}$.

As medições foram realizadas em três drenos do Aterro Sanitário Metropolitano Oeste de Caucaia - CE (ASMOC). Para cada medição, foi empregado um tempo de $20 \mathrm{~min}$, independente da massa de zeólita utilizada. Para isto, na medição realizada no dreno 1 , o cartucho de $100 \mathrm{mg}$ foi conectado à mangueira de entrada do GEM 5000. Ao ligá-lo, com auxílio de uma bomba de sucção, o gás é sugado para interior do equipamento onde são feitas as medições, porém, antes passando pela zeólita, na forma de pellets confinados no cartucho. Nos drenos 2 e 3, adotou-se a mesma condição. Ao fim do uso dos cartuchos com $100 \mathrm{mg}$ de zeólitas 4A, empregaram-se os cartuchos com massas de $200 \mathrm{mg}$ e, ao fim desse ciclo, com massas de $400 \mathrm{mg}$.

\section{Modelo de equilíbrio}

O modelo mais utilizado para representar a adsorção em monocamada é o modelo clássico de Langmuir (Equação 2), que normalmente representa bem as isotermas do tipo I. Esse modelo pressupõe a existência de um número bem definido e localizado de sítios de adsorção, todos energeticamente equivalentes, em que apenas uma molécula é adsorvida por sítio, sem qualquer interação com moléculas adsorvidas em sítios de adsorção vizinhos. ${ }^{16} \mathrm{O}$ modelo de Langmuir permite uma extensão direta para descrever a adsorção multicomponente - Langmuir Estendido - (Equação 3). A seguir são apresentadas as equações de Langmuir e Langmuir estendido, respectivamente:

\section{Langmuir}

$$
q=q_{m} \frac{b P}{1+b P}
$$

Em que $q$ é a quantidade da espécie adsorvida a uma determinada pressão parcial, $q_{m}$ é a quantidade máxima adsorvida da espécie em adsorção, $b$ é a força de interação entre o adsorvente e o adsorbato e $P$ é a pressão parcial do componente em adsorção:

Langmuir Estendido

$$
q=\frac{q_{m, i} b_{i} P_{i}}{1+\left(\sum_{i=1}^{N} b_{i} P_{i}\right)}
$$

Em que $\mathrm{q}_{\mathrm{m}, \mathrm{i}}$ é o valor da quantidade máxima adsorvida da espécie $i ; \mathrm{b}_{\mathrm{i}}$ é relacionado com a afinidade da espécie $i$ pelo adsorbente; $\mathrm{P}_{\mathrm{i}} \mathrm{e}$ é a pressão parcial do componente $i$ na mistura.

O modelo de Sips foi proposto por Sips em 1948 para resolver um problema do aumento contínuo da capacidade de adsorção com o aumento da pressão, sendo uma combinação dos modelos de Langmuir 
e de Freundlich. ${ }^{16,1} \mathrm{O}$ modelo prevê o comportamento heterogêneo da energia na superfície do adsorvente, representada pelo parâmetro $n$. Quando a superfície do adsorvente é considerada homogênea, o modelo de Sips se reduz a Langmuir. As Equações 4 e 5 demonstram o modelo de Sips e sua versão estendida, respectivamente.

$$
\begin{gathered}
q=\frac{q_{\max }(b P)^{1 / n}}{1+(b P)^{1 / n}} \\
q=\frac{q_{\max , i}\left(b_{i} P_{i}\right)^{1 / n_{i}}}{1+\sum_{j=1}^{N}\left(b_{j} P_{j}\right)^{1 / n_{j}}}
\end{gathered}
$$

$n_{i}$ apresenta os efeitos da heterogeneidade energética.

A seletividade $\mathrm{CO}_{2} / \mathrm{CH}_{4}$ foi determinada a partir da quantidade molar obtida através dos modelos de ajustes de Langmuir e Sips Estendido (Equação 6). Também foi calculada a partir dos dados experimentais das isotermas monocomponete de $\mathrm{CO}_{2} \mathrm{e} \mathrm{CH}_{4}$.

$$
S_{\mathrm{CO}_{2} / \mathrm{CH}_{4}}=\frac{q_{\mathrm{CO}_{2}}}{q_{\mathrm{CH}_{4}}} \frac{y_{\mathrm{CH}_{4}}}{y_{\mathrm{CO}_{2}}}
$$

Sendo $q_{\mathrm{CO}_{2}}$ e $q_{\mathrm{CH}_{4}}$ as quantidades molares de cada gás, e $y_{\mathrm{CO}_{2}} \mathrm{e}$ $y_{\mathrm{CH}_{4}}$ as frações molares dos gases na mistura.

\section{RESULTADOS E DISCUSSÃO}

\section{Caracterização da zeólita 4A}

Na Figura 2 é mostrado o difratograma de raios-X da zeólita obtida, o qual apresenta picos bem definidos com boa concordância de localização e intensidade em relação ao padrão da zeólita A (LTA). ${ }^{18}$

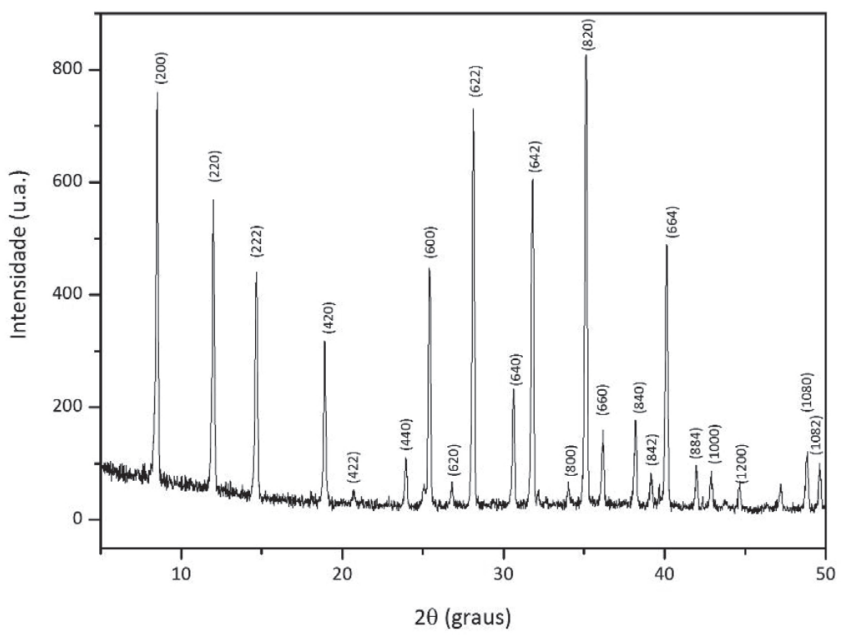

Figura 2. Difratograma de raios- $X$ da zeólita $4 A$ sintetizada

Apenas a fase cristalina correspondente à zeólita 4A foi identificada. A não verificação de outras fases cristalinas indica eficiência no processo de síntese e é importante tendo em vista que a pureza do material confere segurança quanto à reprodutibilidade esperada de determinadas propriedades, como é o caso da sua capacidade em reter $\mathrm{CO}_{2}$ na mistura estudada.

A microscopia eletrônica de varredura foi utilizada como ferramenta auxiliar no estudo da estrutura em microescala da zeólita sintetizada, bem como da sua morfologia. Micrografias da zeólita 4A sintetizada são apresentadas na Figura 3.

Foram obtidas micrografias em diferentes regiões e com diferentes

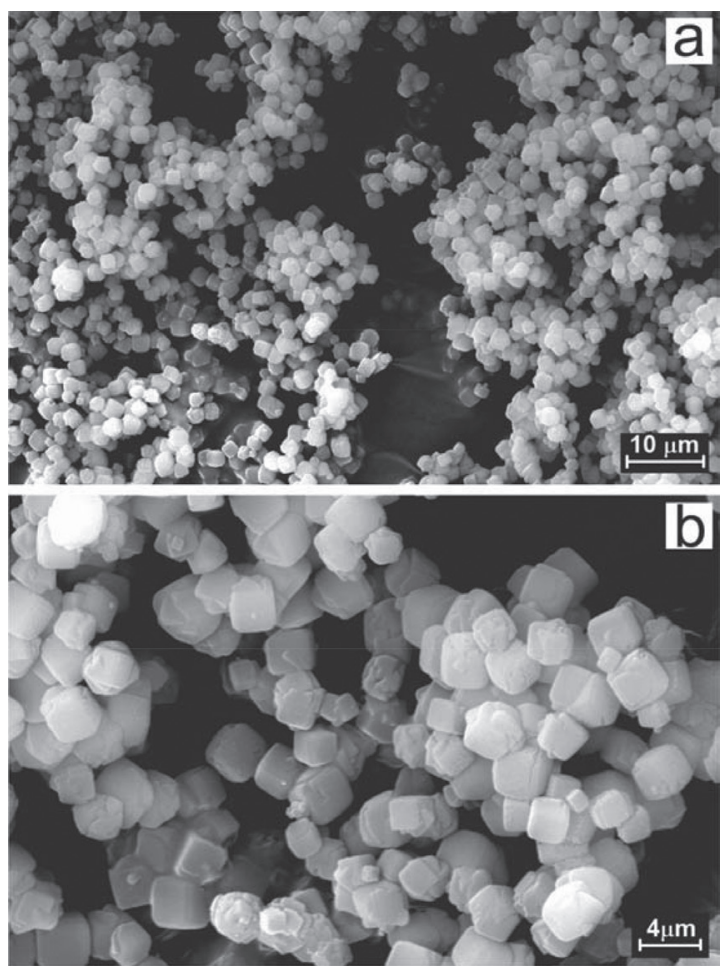

Figura 3. Micrografias (MEV) da zeólita 4A com diferentes magnificações: (a) visão geral mostrando a monodispersividade dos cristais e (b) melhor detalhamento

ampliações. Nelas é possível verificar a existência de cristais com hábito cúbico com forma e tamanhos regulares, características típicas da zeólita A. Os cristais apresentam faces lisas com uma ou outra incrustação, porém, sem variações que possam indicar defeitos ou baixa cristalização. Estas propriedades estão de acordo com o bom grau de cristalização evidenciado por meio da técnica de difração de raios-X.

\section{Adsorção de $\mathrm{CO}_{2}$ em mistura $\mathrm{CO}_{2} / \mathrm{CH}_{4}$}

As isotermas de adsorção de $\mathrm{CO}_{2}$ e $\mathrm{CH}_{4}$ puros pela zeólita $4 \mathrm{~A}$ são apresentadas na Figura 4, enquanto as isotermas correspondentes para a mistura $50 \% \mathrm{CO}_{2} / 50 \% \mathrm{CH}_{4}$, em volume, são mostradas na Figura 5. Considerou-se, como condição térmica apresentada no

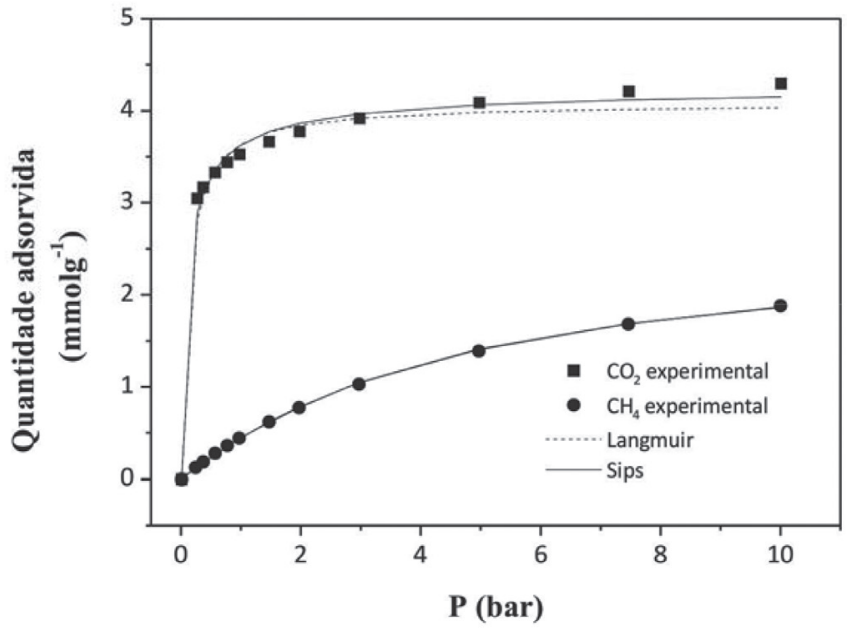

Figura 4. Isotermas de adsorção de $\mathrm{CO}_{2}$ e $\mathrm{CH}_{4}$ a $40{ }^{\circ} \mathrm{C}$. Também são mostrados os ajustes das isotermas experimentais aos modelos de equilíbrio de Langmuir e Sips 

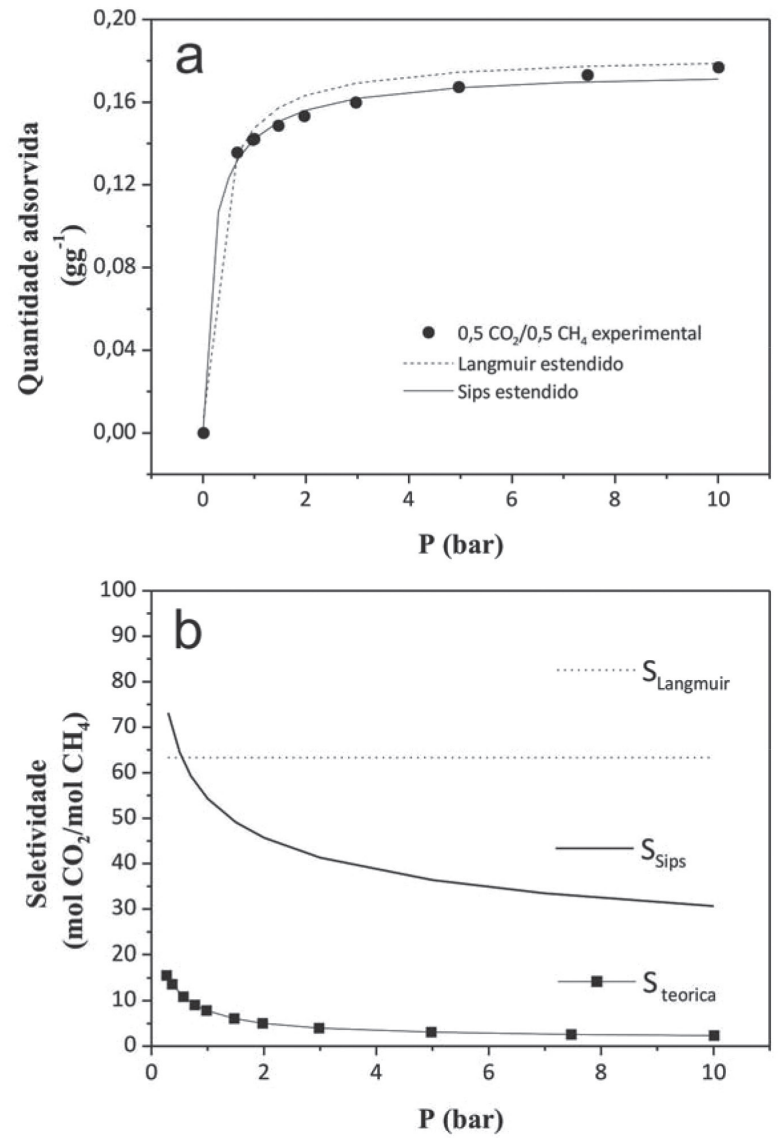

Figura 5. (a) Experimento de adsorção com mistura $50 \% \mathrm{CO}_{2} / 50 \% \mathrm{CH}_{4}$ e predições pelos modelos estendidos de Langmuir e Sips (b) Seletividade em termos de mol $\mathrm{CO}_{2} / \mathrm{mol} \mathrm{CH}_{4}$ calculadas a partir das isotermas monocomponentes e dos modelos estendidos de Langmuir e Sips

aterro sanitário, a temperatura de $40^{\circ} \mathrm{C}$. A zeólita $4 \mathrm{~A}$ foi então testada a $40^{\circ} \mathrm{C}$ sob uma faixa de pressões de 0 a 10 bar.

A isoterma de adsorção de $\mathrm{CO}_{2}$ apresentou forma retangular, indicando alta afinidade a baixas pressões da zeólita pelo $\mathrm{CO}_{2}$. Esta isoterma é típica de materiais zeolíticos e pode ser interessante em processos de captura de $\mathrm{CO}_{2}$. As capacidades de adsorção medidas são comparáveis aos resultados reportados na literatura. ${ }^{19,20}$

A maior capacidade de adsorção de $\mathrm{CO}_{2}$ atribui-se ao fato deste gás apresentar uma maior temperatura crítica quando comparado com $\mathrm{CH}_{4}$. $\mathrm{O}$ dióxido de carbono apresenta comportamento de vapor condensável nas condições estudadas, sendo assim menos volátil e facilmente adsorvido em comparação ao $\mathrm{CH}_{4}$.

Os dados de equilíbrio das isotermas monocomponentes de $\mathrm{CO}_{2}$ e $\mathrm{CH}_{4}$ a $40{ }^{\circ} \mathrm{C}$ foram ajustados aos modelos de equilíbrio de Langmuir e Sips e os parâmetros de ajuste são apresentados na Tabela 1 , encontrando-se valores de $\mathrm{R}^{2}>0,95$ para o ajuste ao modelo de Langmuir e $\mathrm{R}^{2}>0,98$ para o modelo de Sips.

A isoterma da mistura com uma composição de $50 \% \mathrm{CO}_{2}$ e $50 \%$ $\mathrm{CH}_{4}$ (v/v) (Figura 5), junto com a predição dos modelos estendidos de Langmuir e Sips, indica que os dados experimentais são melhor estimados pelo modelo de equilíbrio de Sips estendido no cenário proposto, levando-nos a poder calcular a seletividade em termos de mol $\mathrm{CO}_{2} / \mathrm{mol} \mathrm{CH}_{4}$. A seletividade calculada a partir do modelo de Sips estendido decresceu de $73 \mathrm{~mol}$ para $30 \mathrm{~mol} \mathrm{de} \mathrm{CO}_{2} / \mathrm{mol} \mathrm{CH}_{4}$ enquanto a seletividade teórica encontrada com base nas quantidades adsorvidas de gases diferentes para a mesma pressão de equilíbrio decresceu de $16 \mathrm{~mol} \mathrm{de} \mathrm{CO}_{2} / \mathrm{mol} \mathrm{CH}_{4}$ para 2,3 mol de $\mathrm{CO}_{2} / \mathrm{mol} \mathrm{de}$ $\mathrm{CH}_{4}$ na faixa de pressões estudada. A maior seletividade do $\mathrm{CO}_{2} / \mathrm{CH}_{4}$
Tabela 1. Parâmetros de ajuste dos dados experimentais aos modelos de equilíbrio de Langmuir e Sips

\begin{tabular}{ccccc}
\hline Langmuir & $\mathbf{q}_{\mathbf{m}}\left(\mathbf{m m o l g}^{-1}\right)$ & $\mathbf{b}\left(\mathbf{b a r}^{-1}\right)$ & $\mathbf{n}$ & $\mathbf{R}^{2}$ \\
\hline $\mathrm{CO}_{2}$ & 4,06 & 8,11 & - & 0,95691 \\
$\mathrm{CH}_{4}$ & 2,89 & 0,18 & - & 0,99812 \\
\hline Sips & $\mathbf{q}_{\mathbf{m}}\left(\mathbf{m m o l g}^{-1}\right)$ & $\mathbf{b}\left(\mathbf{b a r}^{-1}\right)$ & $\mathbf{n}$ & $\mathbf{R}^{2}$ \\
\hline $\mathrm{CO}_{2}$ & 4,28 & 9,78 & 1,33 & 0,98223 \\
$\mathrm{CH}_{4}$ & 2,89 & 0,18 & 1 & 0,99812 \\
\hline
\end{tabular}

na mistura em relação à seletividade teórica a partir das isotermas monocomponetes é um indicativo de que o material é fortemente seletivo para o $\mathrm{CO}_{2}$.

\section{Medições gasosas em campo}

Na Figura 6 são apresentadas as curvas de adsorção de $\mathrm{CO}_{2} \mathrm{em}$ função do tempo para as medidas realizadas em campo, em três drenos distintos.
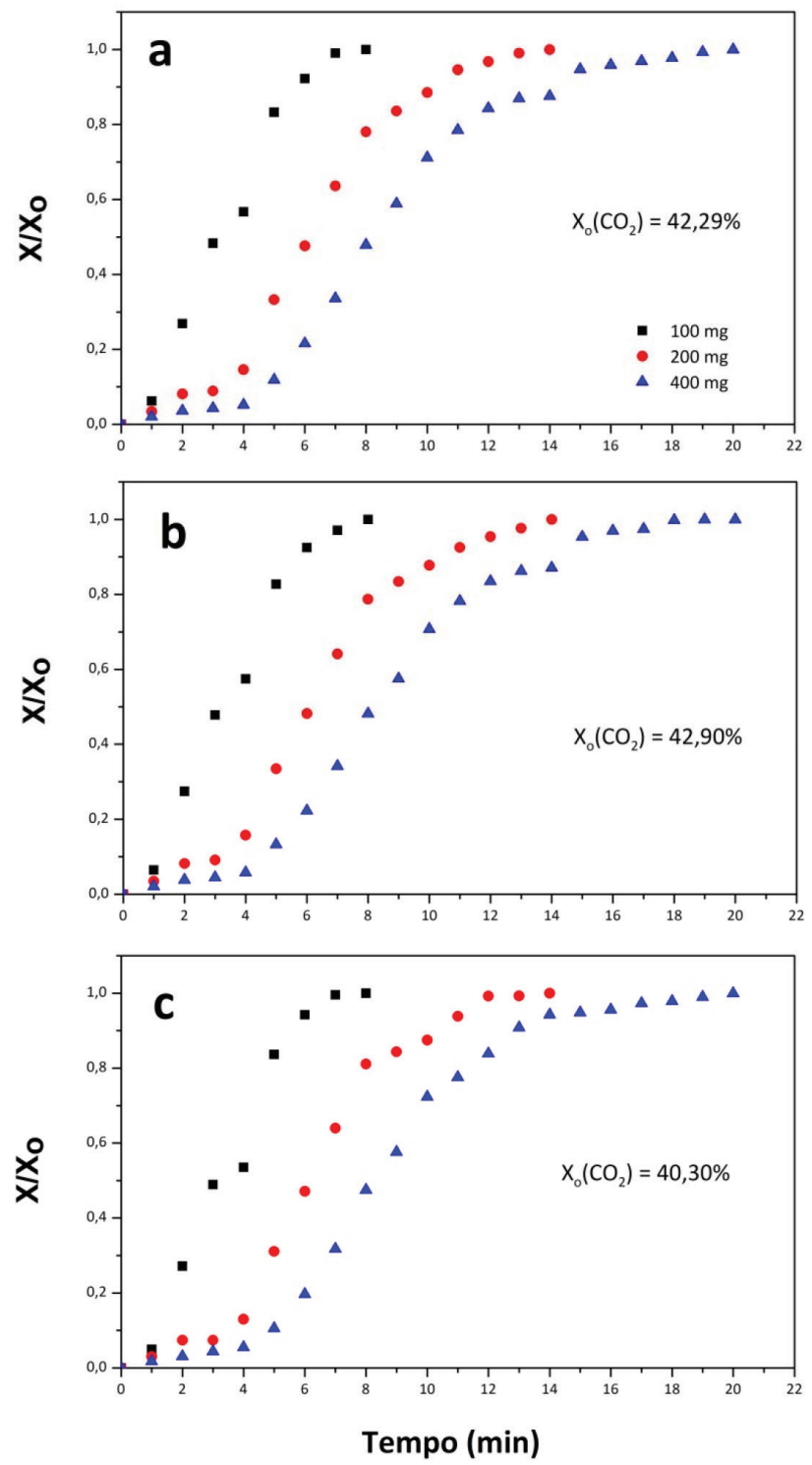

Figura 6. Medições realizadas no (a) dreno $1\left(\mathrm{CO}_{2}=42,29 \%\right)$, (b) dreno 2 $\left(\mathrm{CO}_{2}=42,90\right)$ e (c) dreno $3\left(\mathrm{CO}_{2}=40,30 \%\right)$ 
Os resultados mostram que o teor de $\mathrm{CO}_{2}$ mantém-se sem variações significativas nos diferentes drenos, com pouco mais de $40 \%$. Observa-se o comportamento dos cartuchos de zeólitas quanto à retenção de $\mathrm{CO}_{2}$ presente na mistura do gás de aterro. Para os cartuchos contendo $100 \mathrm{mg}$ de zeólita, observa-se que são necessários aproximadamente $8 \mathrm{~min}$ para a saturação destes. Os tempos de saturação dos demais cartuchos aumentaram, como esperado, proporcionalmente em relação às massas de zeólita utilizadas, sendo necessários cerca de 14 min para os cartuchos contendo 200 mg de zeólita e de 20 min para os cartuchos com $400 \mathrm{mg}$ de zeólita. Convém ressaltar que, do ponto de vista experimental, as medidas realizadas em campo são muito mais complexas que os ensaios realizados em laboratório. No entanto, mesmo não sendo possível um controle tão rigoroso em relação a, por exemplo, vazão dos gases e concentrações dos seus componentes, é possível constatar a eficiência dos materiais nanoporosos no que tange à sua capacidade em reter dióxido de carbono. Tais resultados indicam claramente o potencial de cartuchos da zeólita A na forma de pellets como uma alternativa barata e promissora para a purificação do biogás de aterro sanitário com vistas a seu uso para fins diversos.

\section{CONCLUSÃO}

A zeólita 4A, na forma de pellets em colunas de quartzo, mostrou elevado potencial no processo de purificação do biogás de aterro no que diz respeito à retenção de $\mathrm{CO}_{2}$ e consequente purificação do $\mathrm{CH}_{4}$. A preservação dos resultados obtidos em campo quando comparado aos observados em laboratório indicam que o uso em larga escala desta zeólita, na forma de cartuchos como demonstrado neste trabalho, pode ser uma ferramenta real no que tange o aproveitamento industrial do metano presente nesse tipo de biogás.

\section{AGRADECIMENTOS}

Os autores agradecem à Central Analítica da Universidade Federal do Ceará (UFC/CT-INFRA/MCTI-SISNANO/Pró-equipamentosCAPES) pelo suporte técnico e instrumental das medidas de microscopia de varredura eletrônica, ao Laboratório de Raios-X da
Universidade Federal do Ceará e ao CNPq (processo 402561/2007-4) e à CAPES pelo auxílio financeiro concedido.

\section{REFERÊNCIAS}

1. Skjanes, K.; Rebours, C.; Lindblad, P.; Crit. Rev. Biotechnol. 2013, 2 , 172.

2. Lakaniemie, A. M.; Tuovinen, O. H.; Puhakka J. A.; Bioresour. Technol. 2013, 135, 222.

3. Morishige, K.; J. Phys. Chem. C 2011, 115, 9713.

4. Masoud, M.; Fatemeh, G.; Micropor. Mesopor. Mater. 2014, $200,1$.

5. Oddy, S.; Poupore, J.; Handan, T.; J. Chem. Eng. 2013, 91, 1031.

6. ACS Symposium Series, Flank, W. H., eds.; American Chemical Society: Washington, 1980, n. 135.

7. Melo, D. M. A.; De Souza, J. R.; Melo, M. A. F.; Martinelli, A. E.; Cachima, G. H. B.; Cunha, J. D.; Colloids Surf., A 2006, 272, 32.

8. Jensen, N. K.; Rufford, T. E.; Watson, G.; Zhang, D. K.; Chan, K. I.; May, E. F.; J. Chem. Eng. Data 2012, 57, 106.

9. Yang, R. T.; Adsorbents: fundamentals and applications, John Willey and Sons: New Jersey, 2003.

10. Morishige, K.; J. Phys. Chem. 2011, 115, 9713.

11. Moliner, M.; Martínez, C.; Corma, A.; Angew. Chem., Int. Ed. 2015, 54, 3560 .

12. Barrer, R. M.; Zeolites 1981, 1, 130.

13. Ruthven, D. M.; Tezel, F. H.; Devgun, J. S.; Sridhar, T. S.; Can. J. Chem. Eng. 1984, 62, 526

14. Thompson, R.; Huber, M.; J. Cryst. Growth 1982, 56, 711.

15. Dreisbach, F.; Staudt, R.; Keller, J. U.; Adsortion 1999, 5, 215.

16. Do, D. D.; Ser. Chem. Eng. 1998, 2, 913.

17. Sips, R.; J. Chem. Phys. 1948, 16, 490.

18. Gramlich, V.; Meier, W. M.; Z. Kristallogr. Kristallgeom. Kristallphys. Kristallchem. 1971, 133, 134.

19. Moura, P. A. S.; Bezerra, D. P.; Vilarrasa-Garcia, E.; Bastos-Neto, M.; Azevedo, D. S. C.; Adsorption 2016, 22, 71.

20. Pour, A. A.; Sharifnia, S.; Salehi, R. N.; Ghodrati, M.; J. Nat. Gas Sci. Eng. 2016, 36, 630 